九州大学学術情報リポジトリ

Kyushu University Institutional Repository

\title{
A Preliminary Analysis of Space-Time Structure of Animal Growth by the Use of 4-Dimensional Vector
}

Shimojo, Masataka

Department of Bioresource Sciences, Faculty of Agriculture, Kyushu University

Nakano, Yutaka

University Farm, Faculty of Agriculture, Kyushu University

https://doi.org/10.5109/1467632

出版情報：九州大学大学院農学研究院紀要. 59 (2)，pp.297-299，2014-08-29. Faculty of Agriculture, Kyushu University

バージョン：

権利関係 : 


\title{
A Preliminary Analysis of Space-Time Structure of Animal Growth by the Use of 4-Dimensional Vector
}

\section{Masataka SHIMOJO* and Yutaka NAKANO ${ }^{1}$}

Laboratory of Regulation in Metabolism and Behavior, Division of Animal and Marine Bioresource Sciences, Department of Bioresource Sciences, Faculty of Agriculture, Kyushu University, Fukuoka 812-8581, Japan

(Received April 25, 2014 and accepted May 12, 2014)

\begin{abstract}
This study was designed to make a preliminary analysis of a space-time structure of animal growth by the use of 4-dimensional vector $\left[\boldsymbol{G}=\left(x_{\mathbf{0}}, x_{\mathbf{1}}, x_{\mathbf{2}}, x_{\mathbf{3}}\right)\right]$. The magnitude of $\boldsymbol{G}(G m)$ was regarded as the spacetime structure of the growth of the individual animal. The analytic description of the animal growth was given by $G m$ and its partial differentiation, and the geometric description was given by $G m$ and the cosine component of $\boldsymbol{G}$. Hypotheses (A) (E) were suggested. (A) The space-time dimensional inconsistency was hidden by an invariant that was given by analytic and geometric descriptions of the animal growth. (B) The animal growth was distributed among 4-dimensional space-time axes. (C) The increase in the growth along space axes caused the decrease in the growth along time axis, and vice versa. (D) There was a growth along time axis even when there was no growth along space axes. (E) The geometric description showed that the 4-dimensional information was projected onto 2-dimensional planes and that space and time were related on the complex plane that existed at every point of the space-time structure. This animal growth model suggested a hypothesis that the 4-dimensional space-time structure was related to a pseudo-relativistic growth mechanics and to the complex number world.
\end{abstract}

Key words: Analytic description, animal growth, 4-dimensional vector, geometric description, space-time structure

\section{INTRODUCTION}

The individual animal grows with the passage of time. The growth actually occurs along space axes with the passage of time. Shimojo and Nakano (2014), in a model study, applied 4-dimensional space-time vector to the analysis of the animal growth, where the growth was investigated along both space axes and time axis. In that report, however, the space-time structure was discussed only a little and a mistake has also been found.

This study was designed to make a preliminary analysis of a space-time structure of the animal growth by the use of 4-dimensional vector, and in addition the previous mistake was corrected.

\section{SPACE-TIME STRUCTURE OF ANIMAL GROWTH}

\section{4-dimensional vector for the description of animal growth}

The growth of an individual animal is described using 4-dimensional space-time vector $(\boldsymbol{G})$,

$$
\boldsymbol{G}=\left(x_{0}, x_{1}, x_{2}, x_{3}\right)
$$

where $x_{\mathbf{0}}=$ time (day), $x_{\mathbf{1}}=$ width $(\mathrm{cm}), x_{\mathbf{2}}=\operatorname{depth}(\mathrm{cm})$, $x_{3}=$ height $(\mathrm{cm})$.

${ }^{1}$ University Farm, Faculty of Agriculture, Kyushu University, Kasuya, Fukuoka 811-2307

Part of this study was conducted at Agri-Bio Research Laboratory on Ito Campus, Kyushu University, Motooka, Fukuoka 819-0395

* Corresponding Author (E-mail: mshimojo@agr.kyushu-u.ac.jp)
These units are only one example, and there is a problem of the space-time dimensional inconsistency. The 4-dimensional space-time structure of the animal growth is hypothetically given by the magnitude of $\boldsymbol{G}$ $(G m)$,

$$
G m=\sqrt{\sum_{k=0}^{3} x_{k}^{2} .}
$$

\section{Analytic description of animal growth by the use of 4-dimensional vector}

The animal growth along each of space-time axes is given by $G m$ and its partial differentiation. This leads to an invariant (3). Thus,

$$
\begin{aligned}
& G m \cdot \frac{\partial G m}{\partial x_{0}}=x_{0}, \\
& G m \cdot \frac{\partial G m}{\partial x_{1}}=x_{1}, \\
& G m \cdot \frac{\partial G m}{\partial x_{2}}=x_{2}, \\
& G m \cdot \frac{\partial G m}{\partial x_{3}}=x_{3}, \\
& \left(\frac{\partial G m}{\partial x_{0}}\right)^{2}+\left(\frac{\partial G m}{\partial x_{1}}\right)^{2}+\left(\frac{\partial G m}{\partial x_{2}}\right)^{2}+\left(\frac{\partial G m}{\partial x_{3}}\right)^{2}=1 .
\end{aligned}
$$

This invariant (3) seems to suggest hypotheses (I) 
(III). (I) The space-time dimensional inconsistency is hidden by the mathematical operation (square calculation) giving the invariant (3). (II) The animal growth is distributed among 4-dimensional space-time axes. Generally speaking, the young animal shows a greater growth along space axes, and the old animal shows a greater growth along time axis. (III) The increase in the growth along space axes causes the decrease in the growth along time axis, and vice versa. There is a growth along time axis even when there is no growth along space axes. Do hypotheses (I) (III) seem to suggest a pseudo-relativistic growth mechanics?

The sum of the growth force (4-dimensional growth $(\mathrm{Gm}) \times$ growth acceleration) along each of space-time axes gives

$$
\sum_{\boldsymbol{k}=\mathbf{0}}^{\mathbf{3}}\left(G m \cdot \frac{\partial^{2} G m}{\partial x_{\boldsymbol{k}}^{2}}\right)=3\left(\sum_{\boldsymbol{k}=\mathbf{0}}^{\mathbf{3}}\left(\frac{\partial G m}{\partial x_{\boldsymbol{k}}}\right)^{2}\right)=3 .
$$

This invariant (4) seems to suggest a hypothesis that the growth along each axis is related to the growth along the other 3 axes.

\section{Geometric description of animal growth by the use of 4-dimensional vector}

The animal growth along each of space-time axes is given by $G m$ and the cosine component of $\boldsymbol{G}$. This leads to an invariant (6). Thus,

$$
\begin{aligned}
& G m \cdot \cos \theta_{0}=x_{0}, \\
& G m \cdot \cos \theta_{1}=x_{1}, \\
& G m \cdot \cos \theta_{2}=x_{2}, \\
& G m \cdot \cos \theta_{3}=x_{3}, \\
& \cos ^{2} \theta_{0}+\cos ^{2} \theta_{1}+\cos ^{2} \theta_{2}+\cos ^{2} \theta_{3}=1,
\end{aligned}
$$

where $\theta_{\mathbf{0}}=$ the angle between $\boldsymbol{G}$ and $x_{\mathbf{0}}$-axis (time), $\theta_{\mathbf{1}}$ = the angle between $\boldsymbol{G}$ and $x_{1}$-axis (width), $\theta_{\mathbf{2}}=$ the angle between $\boldsymbol{G}$ and $x_{\mathbf{2}}$-axis (depth), $\theta_{\mathbf{3}}=$ the angle between $\boldsymbol{G}$ and $x_{3}$-axis (height).

This invariant (6) seems to suggest hypotheses (i) (iii). (i) The space-time dimensional inconsistency is hidden by the square calculation giving the invariant (6). (ii) If the animal shows a greater growth along a particular axis among 4-dimensional space-time axes, then the vector $\boldsymbol{G}$ leans more greatly toward that axis. (iii) Generally speaking, the vector $\boldsymbol{G}$ leans toward space axes in young animals, but it leans toward time axis in old animals.

\section{Relationships between analytic description and geometric description of animal growth}

Expression (7) shows the equality between analytic description and geometric description of the animal growth by the use of 4-dimensional space-time vector,

$$
\sum_{\boldsymbol{k}=\mathbf{0}}^{3}\left(\frac{\partial G m}{\partial x_{\boldsymbol{k}}}\right)^{2}=\sum_{\boldsymbol{k}=\mathbf{0}}^{\mathbf{3}}\left(\cos ^{2} \theta_{\boldsymbol{k}}\right)=1
$$

This invariant (7) might be applied to many cases of the animal growth affected by various factors, such as species, sexes, ages, nutritional conditions, natural environmental factors, and social environmental factors.

\section{Projective relationships between 4 dimensions and} 2 dimensions in the geometric description

The geometric description of the animal growth (8) is transformed as follows,

$$
\begin{aligned}
1 & =\cos ^{2} \theta_{0}+\cos ^{2} \theta_{1}+\cos ^{2} \theta_{2}+\cos ^{2} \theta_{3}, \\
& =\cos ^{2} \theta_{0}+\sin ^{2} \theta_{0}, \\
& =\cos ^{2} \theta_{1}+\sin ^{2} \theta_{1}, \\
& =\cos ^{2} \theta_{2}+\sin ^{2} \theta_{2}, \\
& =\cos ^{2} \theta_{3}+\sin ^{2} \theta_{3},
\end{aligned}
$$

where

$$
\begin{aligned}
& \sin ^{2} \theta_{0}=\cos ^{2} \theta_{1}+\cos ^{2} \theta_{2}+\cos ^{2} \theta_{3}, \\
& \sin ^{2} \theta_{1}=\cos ^{2} \theta_{2}+\cos ^{2} \theta_{3}+\cos ^{2} \theta_{0}, \\
& \sin ^{2} \theta_{2}=\cos ^{2} \theta_{3}+\cos ^{2} \theta_{0}+\cos ^{2} \theta_{1}, \\
& \sin ^{2} \theta_{3}=\cos ^{2} \theta_{0}+\cos ^{2} \theta_{1}+\cos ^{2} \theta_{2} .
\end{aligned}
$$

Expressions (8) (9-3) seem to suggest a hypothesis that the 4-dimensional information is projected onto 2-dimensional planes. This hypothesis (projection onto 2-dimensional planes) seems to be applied also to $n$ dimensions and to infinite dimensions.

\section{Space-time relationships on the complex plane}

Since space and time have a degree of 1 each [expression (1)], expressions (8-0) (9-3) with a degree of 2 will be transformed into the expressions with a degree of 1 by factorization. Thus,

$$
\begin{aligned}
1 & =\cos ^{2} \theta_{0}+\sin ^{2} \theta_{0} \\
& =\left(\cos \theta_{0}+\boldsymbol{i} \sin \theta_{0}\right)\left(\cos \theta_{0}-\boldsymbol{i} \sin \theta_{0}\right), \\
& =\cos ^{2} \theta_{1}+\sin ^{2} \theta_{1} \\
& =\left(\cos \theta_{1}+\boldsymbol{i} \sin \theta_{1}\right)\left(\cos \theta_{1}-\boldsymbol{i} \sin \theta_{1}\right), \\
& =\cos ^{2} \theta_{2}+\sin ^{2} \theta_{2} \\
& =\left(\cos \theta_{2}+\boldsymbol{i} \sin \theta_{2}\right)\left(\cos \theta_{2}-\boldsymbol{i} \sin \theta_{2}\right), \\
& =\cos ^{2} \theta_{3}+\sin ^{2} \theta_{3}
\end{aligned}
$$




$$
=\left(\cos \theta_{3}+\boldsymbol{i} \sin \theta_{3}\right)\left(\cos \theta_{3}-\boldsymbol{i} \sin \theta_{3}\right),
$$

where $\boldsymbol{i}$ = imaginary unit,

$$
\begin{aligned}
& \sin \theta_{0}=\sqrt{\cos ^{2} \theta_{1}+\cos ^{2} \theta_{2}+\cos ^{2} \theta_{3}}, \\
& \sin \theta_{1}=\sqrt{\cos ^{2} \theta_{2}+\cos ^{2} \theta_{3}+\cos ^{2} \theta_{0}}, \\
& \sin \theta_{2}=\sqrt{\cos ^{2} \theta_{3}+\cos ^{2} \theta_{0}+\cos ^{2} \theta_{1}}, \\
& \sin \theta_{3}=\sqrt{\cos ^{2} \theta_{0}+\cos ^{2} \theta_{1}+\cos ^{2} \theta_{2}} .
\end{aligned}
$$

Expressions (10-0) (12-3) seem to suggest a hypothesis that space and time are related on the complex plane that exists at every point of the space-time structure.

\section{Animal growth over the interval $t_{1}$ to $t_{2}$}

The space-time vector $\boldsymbol{G}$ and its magnitude $G m$ will be described as follows when applied to the growth over the interval $t_{1}$ to $t_{2}$,

$$
\begin{aligned}
& \Delta \boldsymbol{G}=\left(\Delta x_{0}, \Delta x_{1}, \Delta x_{2}, \Delta x_{3}\right), \\
& \Delta G m=\sqrt{\sum_{k=0}^{3} \Delta x_{k}^{2} .}
\end{aligned}
$$

Correction of the mistake found in the previous report (Shimojo and Nakano, 2014)

The correction is given by replacing 'time' with 'space' in the following sentence appearing in both abstract and text of the previous report (Shimojo and Nakano, 2014). 'If $\boldsymbol{G}$ inclined greatly to $t$-axis compared with space axes $(x, y, z)$, then this was a result of the fact that the animal showed a smaller growth because of the inefficient utilization of time.' (correction: time $\rightarrow$ space)

\section{Conclusions}

This animal growth model suggests a hypothesis that the 4-dimensional space-time structure is related to a pseudo-relativistic growth mechanics and to the complex number world. However, the hypotheses suggested in this report should be severely criticized.

\section{REFERENCES}

Shimojo, M. and Y. Nakano 2014 Pseudo-relativistic vector analysis of animal growth in four-dimensional space-time - Preliminary report with problem of dimensional inconsistency between space and time - J. Fac. Agr., Kyushu Univ., 59: 91-92 